\title{
DEFORMATIONS OF LAGRANGIAN SUBVARIETIES OF HOLOMORPHIC SYMPLECTIC MANIFOLDS
}

\author{
CHRISTIAN LEHN
}

\begin{abstract}
We generalize Voisin's theorem on deformations of pairs of a symplectic manifold and a Lagrangian submanifold to the case of Lagrangian normal crossing subvarieties. Partial results are obtained for arbitrary Lagrangian subvarieties. We apply our results to the study of singular fibers of Lagrangian fibrations.
\end{abstract}

\section{Contents}

Introduction 1

Notations and conventions $\quad 4$

1. Projectivity of Lagrangian subvarieties 4

2. Deformations of symplectic manifolds and Lagrangian subvarieties 6

3. Normal crossing subvarieties 9

4. Codimension formula 12

5. Applications to Lagrangian fibrations 15

$\begin{array}{ll}\text { References } & 19\end{array}$

\section{INTRODUCTION}

In [Vo92] Voisin studied deformations of pairs $Y \subset X$ where $X$ is an irreducible symplectic manifold and $Y$ a complex Lagrangian submanifold. She showed that, roughly speaking, deformations of $X$ where $Y$ stays a complex submanifold are exactly those deformations, where $Y$ stays Lagrangian. We generalize Voisin's results to Lagrangian subvarieties with normal crossings. Let $\pi: \mathfrak{X} \rightarrow M=\operatorname{Def}(X)$ be the universal deformation of $X$. By the Bogomolov-Tian-Todorov theorem, see [Bog78, Tia87, Tod89], we know that $M$ is smooth. Let $\omega \in R^{2} \pi_{*} \mathbb{C}_{\mathfrak{X}} \otimes \mathcal{O}_{M}$ be a class restricting to a symplectic form on the fibers of $\pi$. For a subvariety $i: Y \hookrightarrow X$ denote by $\operatorname{Def}^{\text {ft }}(i)$ the base of the universal locally trivial deformation of $i$ and by $p: \operatorname{Def}^{\mathrm{lt}}(i) \rightarrow M$ the forgetful map. Then we have

2010 Mathematics Subject Classification. 53D05, 32G10, 13D10, 14C30.

Key words and phrases. irreducible symplectic manifolds, lagrangian subvarieties, deformations. 
Theorem 4.3. Let $i: Y \hookrightarrow X$ be a normal crossing Lagrangian subvariety in a compact irreducible symplectic manifold $X$, let $\nu: \widetilde{Y} \rightarrow Y$ be the normalization and denote $j=i \circ \nu$. Consider the subspaces

$$
M_{Y}:=\operatorname{im}\left(\operatorname{Def}^{\mathrm{lt}}(i) \stackrel{p}{\longrightarrow} M\right) \text { and } M_{Y}^{\prime}:=\left\{t \in M: j^{*} \omega_{t}=0\right\}
$$

of $M$. Then $M_{Y}^{\prime}=M_{Y}$ and this space is smooth of codimension

$$
\operatorname{codim}_{M} M_{Y}=\operatorname{codim}_{M} M_{Y}^{\prime}=\operatorname{rk}\left(H^{2}(X, \mathbb{C}) \stackrel{j^{*}}{\longrightarrow} H^{2}(\widetilde{Y}, \mathbb{C})\right)
$$

in $M$.

The space $M_{Y}^{\prime}$ can be thought of as parametrizing those deformations for which $Y$ remains Lagrangian. We are especially interested in the space $M_{Y}$; it parametrizes deformations of $X$ such that $Y$ deforms along with it in a locally trivial manner, so in particular, keeping its singularities. We interpret this space as an invariant of the singularities of $Y$. Therefore, considering locally trivial deformations is not a restriction but has a geometric meaning. Note that if $Y$ is smooth, then every deformation is locally trivial. This is why the above theorem is a generalization of [Vo92, 0.1 Théorème].

Many of the intermediate steps in the proof of Theorem 4.3 are essentially as in [Vo92], but for the smoothness of $M_{Y}$ we have to argue differently. For this, we develop ideas of Ran [Ra92Lif], [Ra92Def] by exploiting the interplay between deformation theory and Hodge theory.

This is also where there normal crossing hypothesis comes from. We show in Proposition 3.6 that locally trivial deformations of the Lagrangian subvariety $Y$ inside $X$ are determined by the sheaf $\widetilde{\Omega}_{Y}$. Its relation to Hodge theory is specific to the normal crossing case. Easy examples show that this is no longer the case for other types of singularities, see Example 5.8. The necessary tools to apply Hodge theoretical arguments over an Artinian base were developed in [Le12].

As in [Vo92], we deduce the following

Corollary 4.4. Let $K:=\operatorname{ker}\left(H^{2}(X, \mathbb{C}) \stackrel{j^{*}}{\longrightarrow} H^{2}(\widetilde{Y}, \mathbb{C})\right)$, let $q$ be the Beauville-Bogomolov quadratic form and consider the period domain

$$
Q:=\left\{\alpha \in \mathbb{P}\left(H^{2}(X, \mathbb{C})\right) \mid q(\alpha)=0, q(\alpha+\bar{\alpha})>0\right\}
$$

of $X$. Then the period map $\wp: M \rightarrow Q$ identifies $M_{Y}$ with $\mathbb{P}(K) \cap Q$ locally at $[X] \in M$.

A normal crossing Lagrangian subvariety in a symplectic manifold is quite special: it cannot have more than two local branches, see Lemma 3.2. I am grateful to Claire Voisin for pointing this out. However, these are still the most important degenerations of Lagrangian submanifolds. For example, the majority of singular fibers of Lagrangian fibrations have normal crossings by 
the results of Hwang-Oguiso [HO09]; so our results apply. A considerable part of Theorem 4.3 holds true for arbitrary Lagrangian subvarieties. More precisely, we have

$$
\left(M_{Y}\right)_{\text {red }} \subset M_{Y}^{\prime} \text { and } \operatorname{codim}_{M} M_{Y}^{\prime}=\operatorname{rk}\left(H^{2}(X, \mathbb{C}) \rightarrow H^{2}(\tilde{Y}, \mathbb{C})\right),
$$

so that we can at least bound the codimension of $M_{Y}$, the space we are interested in, from below, see Theorem 2.6. This enables us to deduce results about Lagrangian fibrations.

Theorem 5.7. Let $X$ be an irreducible symplectic manifold and let $f: X \rightarrow$ $B$ be a Lagrangian fibration. Then $X$ can be deformed, keeping the fibration, to an irreducible symplectic manifold $X^{\prime}$ with a Lagrangian fibration $f^{\prime}$ : $X^{\prime} \rightarrow B^{\prime}$ such that outside a codimension 2 subset $Z \subset B^{\prime}$, all singular fibers of $f^{\prime}$ over the complement of $Z$ are of Kodaira type I, II, III or IV.

This result is based on the Kodaira-type classification of singular fibers by Hwang-Oguiso, see section 5 for details. Similar results on Lagrangian fibrations were independently obtained by Justin Sawon [Saw15] by completely different methods.

Furthermore, the projectivity of arbitrary Lagrangian subvarieties of an irreducible symplectic manifold is shown.

Theorem 1.1. Let $i: Y \hookrightarrow X$ be a complex Lagrangian subvariety in an irreducible symplectic manifold. Then $Y$ is a projective algebraic variety.

This is used to apply results from [Le12], but is also interesting in its own right. Again, the statement was known to Voisin in the smooth case.

Let us spend some words about the structure of this article. In section 1 we show that a Lagrangian subvariety in an irreducible symplectic manifold is always projective. Section 2 is basically an adaption of Voisin's results from [Vo92] to our setting. The main new results of this article are contained in sections 3 and 4 . In section 3 we prove smoothness of $\operatorname{Def}^{\text {lt }}(i)$ in case $Y$ has normal crossings using the $T^{1}$-lifting principle. It also enables us to deduce that the canonical map $p: \operatorname{Def}^{\mathrm{lt}}(i) \rightarrow M$ has constant rank in a neighbourhood of the distinguished point, which implies the smoothness of the image $M_{Y}$. Section 4 finally puts together all previous theory to prove Theorem 4.3 along the lines of Voisin's original argument with some additional input from Hodge theory and deformations of normal crossing varieties. We give applications to Lagrangian fibrations in section 5. First, we relate deformations of a singular fiber to deformations of the fibration and then we try to deform away from very singular fibers. Our results can be applied to most types of the general singular fibers of a Lagrangian fibration in the sense of Hwang-Oguiso [HO09]. 


\section{Notations AND CONVENTIONS}

We work over the field $k=\mathbb{C}$ of complex numbers. The term algebraic variety will stand for a separated reduced $k$-scheme of finite type. In particular, a variety may have several irreducible components. Similarly, a complex variety will be a separated reduced complex space. If there is no danger of confusion, we will drop the adjectives algebraic respectively complex. A variety $Y$ of equidimension $n$ is called a normal crossing variety if for every closed point $y \in Y$ there is an $r \in \mathbb{N}_{0}$ such that $\widehat{\mathcal{O}}_{Y, y} \cong k\left[\left[y_{1}, \ldots, y_{n+1}\right]\right] /\left(y_{1} \cdot \ldots \cdot y_{r}\right)$. It is called a simple normal crossing variety if in addition every irreducible component is nonsingular.

Acknowledgements. The large part of this work is the author's thesis. It is a pleasure to thank my advisor Manfred Lehn for his constant support, for suggesting many interesting problems and for thoroughly asking questions. Furthermore, I would like to thank Duco van Straten for some extremely helpful remarks, Yasunari Nagai and Keiji Oguiso for explaining Lagrangian fibrations, Klaus Hulek for helpful discussions on abelian fibrations, JeanPierre Demailly, Daniel Greb, Sam Grushevsky, Dmitry Kaledin, Thomas Peternell, Sönke Rollenske, Justin Sawon, Gerard van der Geer, Claire Voisin and Kang Zuo for valuable discussions. I am also grateful to Justin Sawon for sending me his preprint [Saw15].

While working on this project, I benefited from the support of the DFG through the SFB/TR 45 "Periods, moduli spaces and arithmetic of algebraic varieties" and the DFG research grant Le 3093/1-1.

\section{Projectivity of Lagrangian subvarieties}

Let $X$ be an irreducible symplectic manifold, that is, a compact, simply connected Kähler manifold such that $H^{0}\left(X, \Omega_{X}^{2}\right)=\mathbb{C} \omega$ for a symplectic form $\omega$. A Lagrangian subvariety $i: Y \hookrightarrow X$ is a subvariety of dimension $\frac{\operatorname{dim} X}{2}$ such that $i^{*} \omega \in H^{0}\left(\Omega_{Y}^{2}\right)$ vanishes on $Y^{\text {reg }} \subset Y$.

If $Y \subset X$ is a smooth Lagrangian subvariety, then by an argument of Voisin, $Y$ is projective even if $X$ is only Kähler, see [Cam06, Prop 2.1]. If $Y \subset X$ is a singular Lagrangian subvariety, it is natural to ask whether $Y$ is still projective. The following affirmative answer to this question is used in the proof of our main theorem, but also interesting in its own right. The proof is a careful adaption of Voisin's argument to the singular setting.

Theorem 1.1. Let $i: Y \hookrightarrow X$ be a complex Lagrangian subvariety in an irreducible symplectic manifold. There is a line bundle $L$ on $Y$ such that $\mathrm{c}_{1}(L)=i^{*} \lambda$ for some Kähler class $\lambda$ on $X$. In particular, $Y$ is a projective algebraic variety. 
Proof. Isomorphism classes of line bundles on $Y$ are classified by the group $H^{1}\left(Y, \mathcal{O}_{Y}^{\times}\right)$, see [GR77, Kap $\left.\mathrm{V}, \S 3.2\right]$. This cohomology group appears in the commutative diagram

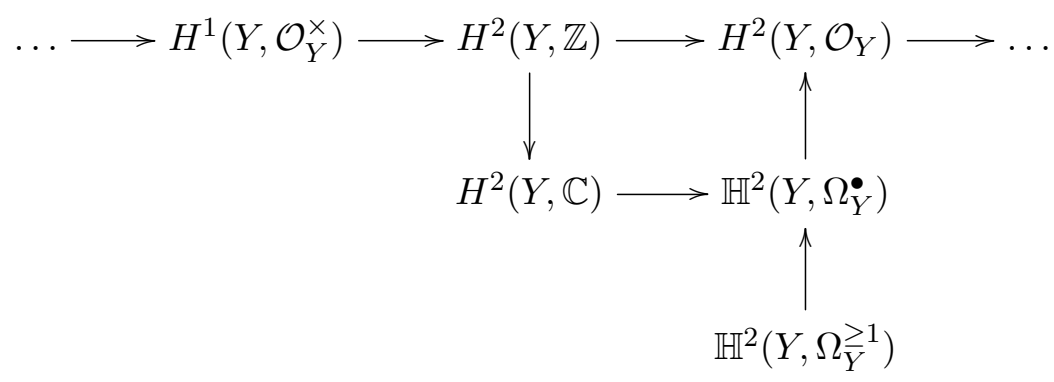

where the first line is the long exact sequence associated with the exponential sequence, see [GR77, Kap V, § 2.4], and the right vertical column comes from the short exact sequence

$$
0 \rightarrow \Omega_{Y}^{\geq 1} \rightarrow \Omega_{Y}^{\bullet} \rightarrow \mathcal{O}_{Y} \rightarrow 0
$$

To obtain a holomorphic line bundle $L$ on $Y$ it is sufficient to find a class $\alpha \in H^{2}(Y, \mathbb{Z})$, such that the image in $\mathbb{H}^{2}\left(Y, \Omega_{Y}^{\bullet}\right)$ comes from $\mathbb{H}^{2}\left(Y, \Omega_{\bar{Y}}^{\geq 1}\right)$. Such $L$ will have $\mathrm{c}_{1}(L)=\alpha$.

As $X$ is Kähler, so is $Y$. Hence, the $H^{k}(Y, \mathbb{Q})$ carry a mixed Hodge structure. Let us consider a resolution of singularities $\pi: \widetilde{Y} \rightarrow Y$. If $W_{m} \subset H^{2}(Y, \mathbb{C})$ denotes the weight filtration, then $\pi^{*}$ factors as

$$
\pi^{*}: H^{2}(Y, \mathbb{C}) \rightarrow H^{2}(Y, \mathbb{C}) / W_{1} \hookrightarrow H^{2}(\widetilde{Y}, \mathbb{C})
$$

As $Y$ is Lagrangian, we have $i^{*} \omega=0$ in $H^{2}(Y, \mathbb{C})$ where $\omega \in H^{0}\left(X, \Omega_{X}^{2}\right)$ is the symplectic form on $X$. Indeed, it maps to 0 in $H^{2}(\widetilde{Y}, \mathbb{C})$ and as $X$ is smooth and morphisms of Hodge structures are strict, $i^{*} \omega$ is in $W_{1}$ if and only if it is zero. Consequently, also $H^{0,2}(X)$ maps to 0 in $H^{2}(Y, \mathbb{C})$.

Let us look at the composition $r: H^{2}(X, \mathbb{C}) \rightarrow H^{2}(Y, \mathbb{C}) \rightarrow \mathbb{H}^{2}\left(Y, \Omega_{Y}^{\bullet}\right)$ and let $H \subset \mathbb{H}^{2}\left(Y, \Omega_{Y}^{\bullet}\right)$ denote the image of $H^{2}(X, \mathbb{R})$. By the Hodgetheoretic considerations above, the image of the Kähler cone $r\left(\mathcal{K}_{X}\right)$ is open in $H$ and clearly, $r\left(H^{2}(X, \mathbb{Q})\right)$ is dense in $H$ so that there is in $0 \neq \alpha^{\prime} \in$ $r\left(\mathcal{K}_{X}\right) \cap r\left(H^{2}(X, \mathbb{Q})\right)$. Then a multiple $\alpha=m \cdot \alpha^{\prime}$ is contained in $r\left(H^{2}(X, \mathbb{Z}) \cap\right.$ $i^{*} r\left(\mathcal{K}_{X}\right)$ and we obtain a line bundle $L$ on $Y$ with the desired property by using the exponential sequence as explained above.

We conclude that $Y$ is projective by [GPR94, Chapter V, Corollary 4.5], see also [Gra62, 3, Satz 1 and Satz 2]. 


\section{Deformations of SYMPLECTIC MANifOLdS AND LAGRANGIAN SUBVARIETIES}

As $H^{0}\left(X, T_{X}\right)=0$ for an irreducible symplectic manifold $X$, the Kuranishi family $\pi: \mathfrak{X} \rightarrow M=\operatorname{Def}(X)$ is universal at the point $0 \in M$ corresponding to $X$. Close to $0 \in M$ the fibers of $\pi$ are again irreducible symplectic manifolds, see [Bea83, §8]. $M$ is known to be smooth by the BogomolovTian-Todorov theorem [Bog78, Tia87, Tod89], see also [GHJ, Thm 14.10]. Therefore, $\operatorname{dim} M=\operatorname{dim} T_{M, 0}=h^{1}\left(T_{X}\right)=h^{1,1}(X)$.

2.1. Deformations of closed immersions. We refer to [Ser06] for an introduction to deformation theory. By $\operatorname{Art}_{k}$ we denote the category of local Artinian $k$-algebras with residue field $k$. Let $i: Y \hookrightarrow X$ be a closed immersion of algebraic $k$-schemes and suppose that $X$ is smooth and proper. Let $R \in \operatorname{Art}_{k}$. A deformation of $i$ over $S=\operatorname{Spec} R$ is a diagram

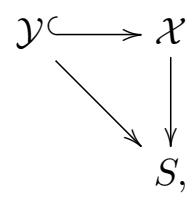

where $\mathcal{X} \rightarrow S$ and $\mathcal{Y} \rightarrow S$ are flat and the fiber of (2.1) over $k=R / \mathfrak{m}_{R}$ is isomorphic to $i: Y \hookrightarrow X$. Such a deformation is locally trivial if for every $x \in X, y \in Y$ with $i(y)=x$ there is an open subset $U \subset X$ and such that $x \in U$ and the restriction $\left.\left.\mathcal{Y}\right|_{U} \hookrightarrow \mathcal{X}\right|_{U}$ is a trivial deformation of $Y \cap U \hookrightarrow U$. The tangent space to the deformation space of locally trivial deformations of a proper $k$-variety $Y$ is given by $H^{1}\left(T_{Y}\right)$, see [Ser06, Proposition 1.2.9]. Let $i: Y \hookrightarrow X$ be a closed immersion with smooth $X$ and let $T_{X}\langle Y\rangle$ be the kernel of the natural map $T_{X} \rightarrow i_{*} N_{Y / X}$, see [Ser06, 3.4.4]. Then the tangent space to the deformation space for locally trivial deformations of $i$ is given by $H^{1}\left(T_{X}\langle Y\rangle\right)$, see [Ser06, Proposition 3.4.17]. More generally, given a diagram like (2.1), we define $T_{\mathcal{X} / S}\langle\mathcal{Y}\rangle$ by the exact sequence of sheaves on $\mathcal{X}$

$$
0 \longrightarrow T_{\mathcal{X} / S}\langle\mathcal{Y}\rangle \longrightarrow T_{\mathcal{X} / S} \longrightarrow N_{\mathcal{Y} / \mathcal{X}}^{\prime} \longrightarrow 0
$$

where

$$
N_{\mathcal{Y} / \mathcal{X}}^{\prime}:=\operatorname{ker}\left(N_{\mathcal{Y} / \mathcal{X}} \rightarrow T_{\mathcal{Y} / S}^{1}\right)
$$

is the equisingular normal sheaf.

Let $i: Y \hookrightarrow X$ be the inclusion of a closed subvariety in an irreducible symplectic manifold. Then, as a consequence of [FK87], there is a universal locally trivial deformation of $i$ over a (germ of a) complex space $\operatorname{Def}^{\mathrm{lt}}(i)$. The inclusion $Y \hookrightarrow X$ gives a point $0 \in \operatorname{Def}^{\mathrm{lt}}(i)$. By construction there is a forgetful morphism $p: \operatorname{Def}^{\text {lt }}(i) \rightarrow M$ of complex spaces with $p(0)=0$. 
Definition 2.2. We denote by $M_{Y} \subset M$ the image of $p$, that is, the smallest closed complex subspace such that $p$ factors through $M_{Y} \hookrightarrow M$.

2.3. The locus where a subvariety is Lagrangian. Let $i: Y \hookrightarrow X$ be the inclusion of a Lagrangian subvariety in an irreducible symplectic manifold $X$ of dimension $2 n$.

We take a flat section $0 \neq \omega \in R^{0} \pi_{*} \Omega_{\mathfrak{X} / M}^{2} \hookrightarrow \mathscr{H}^{2}:=R^{2} \pi_{*} \mathbb{C}_{\mathfrak{X}} \otimes \mathcal{O}_{M}$ and write $\omega_{t}:=\left.\omega\right|_{X_{t}}$ for the symplectic form on the fiber $X_{t}=\pi^{-1}(t)$. We interpret $\omega_{t}$ as an element of $H^{2}(X, \mathbb{C})$. Let $[Y] \in H^{2 n}(X, \mathbb{Z})$ denote the Poincaré dual of the fundamental cycle of $Y$. It has a unique flat lift to $\mathscr{H}^{2}$ and we denote by $[Y]_{t}$ the restriction of this lift to $\mathscr{H}_{t}^{2}=H^{2}\left(X_{t}, \mathbb{C}\right)$. Let us denote by $\nu: \widetilde{Y} \rightarrow Y$ a resolution of singularities and put $j=i \circ \nu$.

Definition 2.4. With these notations following Voisin [Vo92] we define

$$
M_{Y}^{\prime}:=\left\{t \in M \mid j^{*} \omega_{t}=0 \text { in } H^{2}(\widetilde{Y}, \mathbb{C})\right\} .
$$

Clearly, this definition is independent of the resolution $\nu: \widetilde{Y} \rightarrow Y$.

Lemma 2.5. The tangent space of $M_{Y}^{\prime}$ at 0 is given by

$$
T_{M_{Y}^{\prime}, 0}=\operatorname{ker}\left(H^{1}\left(T_{X}\right) \stackrel{\omega^{\prime}}{\longrightarrow} H^{1}\left(\Omega_{X}\right) \stackrel{j^{*}}{\longrightarrow} H^{1}\left(\Omega_{\widetilde{Y}}\right)\right)
$$

where $\omega^{\prime}$ is the isomorphism induced by the symplectic form on $X$.

Proof. Locally at $0 \in M$ the space $M_{Y}^{\prime}$ is cut out by the equation $j_{t}^{*} \omega_{t}=0$. Therefore, the tangent space at 0 is given by the equation

$$
0=\left.\left(\nabla j_{t}^{*} \omega_{t}\right)\right|_{t=0}=\left.j^{*}\left(\nabla \omega_{t}\right)\right|_{t=0},
$$

where $\nabla$ is the Gauß-Manin connection. At 0 it can be identified with the map $H^{0}\left(\Omega_{X}^{2}\right) \rightarrow \operatorname{Hom}\left(H^{1}\left(T_{X}\right), H^{1}\left(\Omega_{X}\right)\right)$ given by cup product and contraction, which concludes the proof.

Theorem 2.6. Let $i: Y \hookrightarrow X$ be a Lagrangian subvariety in a compact irreducible symplectic manifold $X$, let $\nu: \widetilde{Y} \rightarrow Y$ be a resolution of singularities and denote $j=i \circ \nu$. Then $\left(M_{Y}\right)_{\mathrm{red}} \subset M_{Y}^{\prime}$ and $M_{Y}^{\prime}$ is smooth of codimension

$$
\operatorname{codim}_{M} M_{Y}^{\prime}=\operatorname{rk}\left(H^{2}(X, \mathbb{C}) \stackrel{j^{*}}{\longrightarrow} H^{2}(\widetilde{Y}, \mathbb{C})\right)
$$

in $M$.

Proof. First assume that $Y$ is irreducible. Voisin shows in [Vo92, Propositions 1.2 and 1.7] that $M_{Y}^{\prime}$ is a smooth submanifold of $M$ and that it coincides the Hodge locus $M_{[Y]}$ associated with the class $[Y]$ of $Y$ in $H^{2 n}(X, \mathbb{C})$, see [Vo2, Ch 5.3]. Smoothness of $Y$ is not needed for the first proposition, as its proof only uses the class of $Y$. The second one uses [Vo92, Lemme 1.5] which has to be replaced by Lemma 2.7 below. 
Now let $Y=\cup_{i} Y_{i}$ be a decomposition into irreducible components. Then set-theoretically

$$
M_{Y} \subset \bigcap_{i} M_{Y_{i}} \subset \bigcap_{i} M_{\left[Y_{i}\right]}=\bigcap_{i} M_{Y_{i}^{\prime}}=M_{Y}^{\prime},
$$

where the first inclusion is a consequence of [Le12, Lemma 1.4], the inclusion $M_{Y_{i}} \subset M_{\left[Y_{i}\right]}$ is obvious and the equalities follow from the irreducible case and the definition of $M_{Y}^{\prime}$. The statement about the codimension is deduced from the description (2.5) of the tangent space of $M_{Y}^{\prime}$.

The following straight-forward generalization of [Vo92, Lemme 1.5] will complete the proof of Theorem 2.6. We include a full proof for convenience. Let $\mu: H^{2}(X, \mathbb{C}) \rightarrow H^{2+2 n}(X, \mathbb{C})$ be the map given by cup product with $[Y]$ and observe that it factors as $H^{2}(X, \mathbb{C}) \stackrel{j^{*}}{\longrightarrow} H^{2}(\tilde{Y}, \mathbb{C}) \stackrel{j_{*}}{\longrightarrow} H^{2+2 n}(X, \mathbb{C})$.

Lemma 2.7. If $Y$ is irreducible, then

$$
\operatorname{ker}\left(H^{2}(X, \mathbb{C}) \stackrel{\mu}{\longrightarrow} H^{2 n+2}(X, \mathbb{C})\right)=\operatorname{ker}\left(H^{2}(X, \mathbb{C}) \stackrel{j^{*}}{\longrightarrow} H^{2}(\widetilde{Y}, \mathbb{C})\right) .
$$

Proof. We show equality of the respective kernels with real coefficients. From $\mu=j_{*} j^{*}$ we immediately have $\operatorname{ker} j^{*} \subset \operatorname{ker} \mu$. For the other inclusion we choose a Kähler class $\kappa \in H^{2}(X, \mathbb{R})$. We have to show that $j_{*}$ is injective on $\operatorname{im} j^{*}$.

Assume $n=1$. As $\tilde{Y}$ is connected, $H^{2}(\widetilde{Y}, \mathbb{C}) \cong \mathbb{C}$ and the map $j_{*}$ : $H^{2}(\widetilde{Y}, \mathbb{C}) \rightarrow H^{2}(X, \mathbb{C})$ is given by $1 \mapsto[Y]$. As $X$ is Kähler, $[Y] \neq 0$. So $j_{*}$ is injective and the claim follows.

If $n \geq 2$, choose a Kähler class $\kappa \in H^{2}(X, \mathbb{R})$. We may assume that $\widetilde{Y} \rightarrow Y$ is obtained by a sequence of blow-ups in smooth centers. Hence there is a Kähler class of the form $\widetilde{\kappa}=j^{*} \kappa-\sum_{i} \delta_{i} E_{i} \in H^{2}(\widetilde{Y}, \mathbb{R})$ where the $E_{i}$ are exceptional divisors and $\delta_{i} \in \mathbb{Q}$ are positive. We define a bilinear form

$$
q(\alpha, \beta):=\int_{\widetilde{Y}} \widetilde{\kappa}^{n-2} \cdot \alpha \cdot \beta \quad \alpha, \beta \in H^{2}(\widetilde{Y}, \mathbb{C})
$$

on $H^{2}(\widetilde{Y}, \mathbb{C})$. For $\alpha, \beta \in H^{2}(X, \mathbb{R})$ this gives

$$
\begin{aligned}
q\left(j^{*} \alpha, j^{*} \beta\right) & =\int_{\widetilde{Y}} \widetilde{\kappa}^{n-2} \cdot j^{*}(\alpha \cdot \beta)=\int_{X} j_{*}\left(\widetilde{\kappa}^{n-2} \cdot j^{*}(\alpha \cdot \beta)\right) \\
& =\int_{X} \mu\left(\kappa^{n-2}\right) \cdot \alpha \cdot \beta=\int_{X} \kappa^{n-2} \cdot \mu(\alpha) \cdot \beta .
\end{aligned}
$$

So we see that if $\mu(\alpha)=0$, then $q\left(j^{*} \alpha, j^{*} \beta\right)=0$ for all $\beta \in H^{2}(X, \mathbb{R})$. To conclude that $j^{*} \alpha=0$ it would be sufficient to see that $q$ is non-degenerate on $\operatorname{im} j^{*} \subset H^{2}(\widetilde{Y}, \mathbb{R})$. On the whole of $H^{2}(\widetilde{Y}, \mathbb{R})$ the form $q$ is non-degenerate by the Hodge index theorem, see [Vo1, Thm 6.33]. Here we need that $\widetilde{\kappa}$ is a Kähler class. That $q$ remains non-degenerate on the subspace im $j^{*}$ can also be deduced as follows. As $Y$ is Lagrangian $\operatorname{im} j^{*} \subset H^{1,1}(\widetilde{Y}, \mathbb{R}):=H^{1,1}(\widetilde{Y}) \cap$ 
$H^{2}(\tilde{Y}, \mathbb{R})$ and on $H^{1,1}(\tilde{Y}, \mathbb{R})$ the form $q$ is non degenerate and has signature $\left(1, h^{1,1}-1\right)$. We know that $q\left(j^{*} \kappa, j^{*} \kappa\right)>0$ and so $q$ is negative definite on $j^{*} \kappa^{\perp}$. Write $j^{*} \alpha=c \cdot j^{*} \kappa+\alpha^{\prime}$ where $\alpha^{\prime} \in j^{*} \kappa^{\perp}$. The decomposition shows that $\alpha^{\prime} \in \operatorname{im} j^{*}$ as well. Then if $j^{*} \alpha \neq 0$ at least one of the numbers $q\left(j^{*} \alpha, j^{*} \kappa\right), q\left(j^{*} \alpha, \alpha^{\prime}\right)$ is nonzero and so $\mu(\alpha) \neq 0$.

\section{NORMAL CROSSING SUBVARIETIES}

Our next goal is to prove smoothness of the space $\operatorname{Def}^{1 \mathrm{lt}}(i)$ of locally trivial deformations of $i: Y \hookrightarrow X$, see Theorem 3.8, using a variant of the $T^{1}$ lifting principle. Smoothness plays an important role in the proof of our main result, Theorem 4.3. We start with some preliminary considerations on normal crossing varieties.

Definition 3.1. Let $f: \mathcal{Y} \rightarrow S$ be a proper morphism of schemes. We define $\tau_{\mathcal{Y} / S}^{k} \subset \Omega_{\mathcal{Y} / S}^{k}$ to be the subsheaf of sections whose support is contained in the singular locus of $f$. We put $\widetilde{\Omega}_{\mathcal{Y} / S}^{k}:=\Omega_{\mathcal{Y} / S}^{k} / \tau_{\mathcal{Y} / S}^{k}$. Clearly, the exterior differential makes $\widetilde{\Omega}_{\mathcal{Y} / S}^{\bullet}$ into a complex.

If $Y$ is a normal crossing $\mathbb{C}$-variety, then the natural map $\mathbb{C} \rightarrow \widetilde{\Omega}_{Y}^{\bullet}$ is a resolution. Moreover, if $Y$ is proper, this complex can be used to define the mixed Hodge structure on $H^{k}(Y, \mathbb{C})$ as it has been done in [Fri83] if $Y$ has simple normal crossings. For a locally trivial deformation $f: \mathcal{Y} \rightarrow S$ of a simple normal crossing variety over an Artinian base scheme, it has been shown in [Le12] that $\widetilde{\Omega}_{\mathcal{Y} / S}^{\bullet}$ is a resolution of the constant sheaf $f^{-1} \mathcal{O}_{S}$ and the Hodge theoretic analogues of Friedman's results have been established. It is possible to extend these results to the normal crossing case, i.e. components are allowed to have self-intersections. For this, let $Y$ be a normal crossing variety. We need a semi-simplicial resolution $\ldots \rightrightarrows Y^{[1]} \rightrightarrows Y^{[0]} \rightarrow Y$ which replaces the canonical one in the simple normal crossing case, see [Fri83, p. 77] and [Le12, 4.4]. For Lagrangian subvarieties the situation is very simple. I am grateful to Claire Voisin for this observation. Its proof is straightforward, cf. [GLR14, Lemma 5.3].

Lemma 3.2. If $Y \subset X$ is a Lagrangian subvariety with normal crossings in a symplectic manifold $X$, then locally there cannot be more than two components.

Remark 3.3. We thus obtain a semi-simplicial resolution where $\nu: Y^{[0]} \rightarrow Y$ is the normalization, $Y^{[1]}:=\nu^{-1}\left(Y^{\text {sing }}\right)$ and the morphisms $Y^{[1]} \rightrightarrows Y^{[0]}$ are the inclusion and its composition with the canonical involution $\tau: Y^{[1]} \rightarrow$ $Y^{[1]}$ exchanging the two branches. Using this resolution, the Hodge theoretic results from [Le12] carry over to the normal crossing situation. 
Let $\mathcal{X} \rightarrow S=\operatorname{Spec} R$ for $R \in \operatorname{Art}_{k}$ be a deformation of an irreducible symplectic manifold $X$ and let $\omega \in H^{0}\left(\Omega_{\mathcal{X} / S}^{2}\right)$ be a relative symplectic form.

Lemma 3.4. Let $i: Y \hookrightarrow X$ be a normal crossing Lagrangian subvariety. If $\mathcal{Y} \hookrightarrow \mathcal{X}$ is a locally trivial deformation of $i$ over $S$, then $\mathcal{Y}$ is Lagrangian with respect to the symplectic form $\omega$ on $\mathcal{X}$.

Proof. Let $\widetilde{\mathcal{Y}} \rightarrow S$ be the locally trivial deformation of the normalization of $Y$ obtained from [Le12, Lemma 4.5]. Note that $Y$ is projective by Theorem 1.1, so Lemma [Le12, Lemma 4.5] can be applied. As $Y$ has normal crossings, $\tilde{\mathcal{Y}} \rightarrow S$ is smooth and $H^{0}\left(\Omega_{\mathcal{X} / S}^{2}\right)$ and $H^{0}\left(\Omega_{\tilde{\mathcal{Y}} / S}^{2}\right)$ are free $\mathcal{O}_{S}$ modules by [Del68, Théorème 5.5]. Therefore, the pullback $H^{0}\left(\Omega_{\mathcal{X} / S}^{2}\right) \rightarrow H^{0}\left(\Omega_{\widetilde{\mathcal{Y}} / S}^{2}\right)$ has constant rank by [Le12, Theorem 4.17]. As $\operatorname{rk}\left(j^{*} \otimes \mathbb{C}\right)=0$ on the central fiber, $j^{*}$ is identically zero and thus $\mathcal{Y}$ is Lagrangian.

Lemma 3.5. Let $i: Y \hookrightarrow X$ be a Lagrangian subvariety in an irreducible symplectic manifold $X$, let $S=\operatorname{Spec} R$ where $R \in \operatorname{Art}_{\mathbb{C}}$ and let $\mathcal{Y} \hookrightarrow \mathcal{X}$ is a locally trivial deformation of $i$ over $S$. Then the symplectic form $\omega \in$ $H^{0}\left(\Omega_{\mathcal{X} / S}^{2}\right)$ induces a morphism between the exact sequences

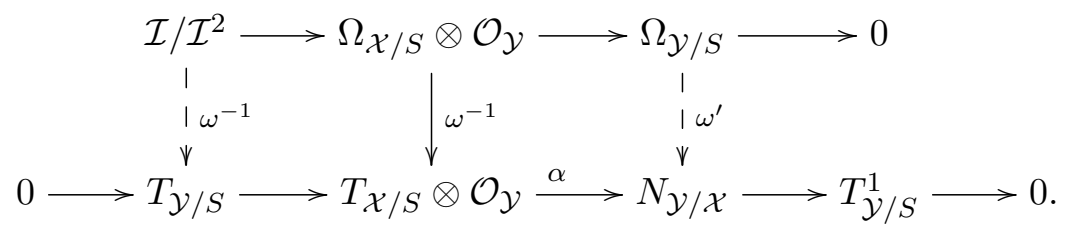

Proof. Since $\omega$ is non-degenerate, the map $\omega^{-1}: \Omega_{\mathcal{X} / S} \rightarrow T_{\mathcal{X} / S}$ is an isomorphism. The composition $\varphi: \mathcal{I} / \mathcal{I}^{2} \rightarrow N_{\mathcal{Y} / \mathcal{X}}=\operatorname{Hom}\left(\mathcal{I} / \mathcal{I}^{2}, \mathcal{O}_{\mathcal{Y}}\right)$ is given by $f \mapsto\{f, \cdot\}$ where $\{\cdot, \cdot\}$ is the Poisson bracket associated with $\omega$. So $\varphi=0$ and the restriction of $\omega^{-1}$ to $\mathcal{I} / \mathcal{I}^{2}$ factors through $T_{\mathcal{Y} / S}=\operatorname{ker} \alpha$. Once we have this, we obtain a morphism $\omega^{\prime}: \Omega_{\mathcal{Y} / S} \rightarrow N_{\mathcal{Y} / \mathcal{X}}$, as the first line of (3.1) is exact, by lifting sections to $\Omega_{\mathcal{X} / S} \otimes \mathcal{O}_{\mathcal{Y}}$.

It is well-known that if in the situation of the preceding lemma the morphism $f: \mathcal{Y} \rightarrow S$ is smooth, then $\omega$ gives an isomorphism $\Omega_{\mathcal{Y} / S} \rightarrow N_{\mathcal{Y} / \mathcal{X}}$. The following Proposition 3.6 explains what happens for singular Lagrangian subvarieties.

Proposition 3.6. Let $i: Y \hookrightarrow X$ be a Lagrangian subvariety in an irreducible symplectic manifold $X$, let $S=\operatorname{Spec} R$ where $R \in \operatorname{Art}_{\mathbb{C}}$ and let $\mathcal{Y} \hookrightarrow \mathcal{X}$ be a locally trivial deformation of $i$ over $S$. Let $\omega^{\prime}: \Omega_{\mathcal{Y} / S} \rightarrow N_{\mathcal{Y} / \mathcal{X}}$ be as in (3.1) and let $N_{\mathcal{Y} / \mathcal{X}}^{\prime}$ be the equisingular normal sheaf defined in (2.3). 
Then the diagram

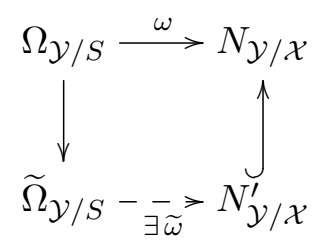

can be completed and $\widetilde{\omega}: \widetilde{\Omega}_{\mathcal{Y} / S} \rightarrow N_{\mathcal{Y} / \mathcal{X}}^{\prime}$ is an isomorphism.

Proof. As $Y$ is Lagrangian, it is of pure dimension and thus $\mathcal{O}_{Y}$ and also $\mathcal{O}_{\mathcal{Y}}$ have no embedded primes. Locally, the sheaf $N_{\mathcal{Y} / \mathcal{X}}$ can be embedded in a locally free sheaf and thus it does not have any embedded primes either. Hence, $\tau_{\mathcal{Y} / S}^{1}$ maps to zero and $\widetilde{\omega}$ exists. But as $\omega$ is an isomorphism at smooth points of $f$, the support of ker $\omega$ is contained in the singular locus of $f$, hence $\operatorname{ker} \omega \subset \tau_{\mathcal{Y} / S}^{k}$ and $\widetilde{\omega}$ is injective. Moreover, $\widetilde{\Omega}_{\mathcal{Y} / S}$ maps onto $\operatorname{ker}\left(N_{\mathcal{Y} / \mathcal{X}} \rightarrow T_{\mathcal{Y} / S}^{1}\right)$ by (3.1), hence is identified with $N_{\mathcal{Y} / \mathcal{X}}^{\prime}$.

3.7. The $T^{1}$-lifting Principle. To prove smoothness of $\operatorname{Def}^{\mathrm{lt}}(i)$ we will use Ran's $T^{1}$-lifting principle [Ra92Def, Kaw92, Kaw97], we refer to [Le11] for a gentle introduction. The basic idea is that in order to prove smoothness of a deformation functor it suffices to show that the corresponding $T^{1}$-modules are locally free for every infinitesimal deformation over a local Artinian scheme. This is achieved by means of Hodge theory.

Theorem 3.8. Let $Y$ be a Lagrangian normal crossing subvariety of an irreducible symplectic manifold $X$. Then the complex space $\operatorname{Def}^{\mathrm{lt}}(i)$ is smooth at 0 . Moreover, $M_{Y}$ is smooth and $\operatorname{Def}^{\mathrm{lt}}(i) \rightarrow M_{Y}$ is a submersion.

Proof. We have to show that the $H^{1}\left(T_{\mathcal{X} / S}\langle\mathcal{Y}\rangle\right)$ are free. The sheaf $T_{\mathcal{X} / S}\langle\mathcal{Y}\rangle$ was defined in (2.2). Let $i: Y \hookrightarrow X$ be the inclusion and let $\mathcal{Y} \hookrightarrow \mathcal{X}$ be a locally trivial deformation of $i$ over $S=\operatorname{Spec} R$ for $R \in \operatorname{Art}_{\mathbb{C}}$. Consider the long exact sequence

$$
0 \rightarrow H^{0}\left(T_{\mathcal{X} / S}\langle\mathcal{Y}\rangle\right) \rightarrow H^{0}\left(T_{\mathcal{X} / S}\right) \rightarrow H^{0}\left(N_{\mathcal{Y} / \mathcal{X}}^{\prime}\right) \rightarrow H^{1}\left(T_{\mathcal{X} / S}\langle\mathcal{Y}\rangle\right) \rightarrow \ldots
$$

obtained from the sequence (2.2). We transform this sequence using the isomorphism $T_{\mathcal{X} / S} \cong \Omega_{\mathcal{X} / S}$, Lemma 3.4, Proposition 3.6 and $H^{0}\left(\Omega_{\mathcal{X} / S}\right)=0$ to obtain an exact sequence

$$
0 \rightarrow H^{0}\left(\widetilde{\Omega}_{\mathcal{Y} / S}\right) \rightarrow H^{1}\left(T_{\mathcal{X} / S}\langle\mathcal{Y}\rangle\right) \rightarrow H^{1}\left(\Omega_{\mathcal{X} / S}\right) \rightarrow H^{1}\left(\widetilde{\Omega}_{\mathcal{Y} / S}\right) \rightarrow \ldots
$$

Recall from Definition 2.2 that we have a factorization $p: \operatorname{Def}^{\text {lt }}(i) \rightarrow$ $M_{Y} \hookrightarrow M$. However, in general it is not clear whether $\operatorname{Def}^{\text {lt }}(i) \rightarrow M_{Y}$ is surjective, let alone submersive. By Theorem [Del68, Théorème 5.5] we 
know that $H^{k}\left(\Omega_{\mathcal{X} / S}\right)$ is free. By Theorem 1.1 we know that $Y$ is a projective variety, so Theorem [Le12, Theorem 4.13] applies and $H^{k}\left(\widetilde{\Omega}_{\mathcal{Y} / S}\right)$ is free. Note that the results of [Le12] carry over literally to the normal crossing case as was explained in Remark 3.3. Then by Theorem [Le12, Theorem 4.22] also the cokernel (and hence the kernel) of $H^{k}\left(\Omega_{\mathcal{X} / S}\right) \rightarrow H^{k}\left(\widetilde{\Omega}_{\mathcal{Y} / S}\right)$ is free. From sequence (3.4) we deduce that all $H^{k}\left(T_{\mathcal{X} / S}\langle\mathcal{Y}\rangle\right)$ are free and that all morphisms in (3.4) have constant rank. In particular, all morphisms in (3.3) have constant rank. The $T^{1}$-lifting principle implies that $\operatorname{Def}^{\mathrm{lt}}(i)$ is smooth.

So the canonical morphism $p:\left(\operatorname{Def}^{\mathrm{lt}}(i), 0\right) \rightarrow(M, 0)$ is just a holomorphic map between (germs of) complex manifolds. To prove the theorem it suffices to show that ts differential $D p$ has constant rank in a neighbourhood of 0 . This holds if the stalk of $\operatorname{coker}\left(p_{*}: T_{\operatorname{Def}^{\mathrm{lt}}(i)} \rightarrow p^{*} T_{M}\right)$ at 0 is free. Freeness may be tested after completion, and then by the local criterion for flatness [Ser06, Thm A.5] we may test it for the truncations modulo powers of the maximal ideal. In other words we have to verify, given as above a locally trivial deformation $\mathcal{Y} \hookrightarrow \mathcal{X}$ of $i$ over $S=\operatorname{Spec} R$ with $R \in \operatorname{Art}_{\mathbb{C}}$, that the map $H^{1}\left(T_{\mathcal{X} / S}\langle\mathcal{Y}\rangle\right) \rightarrow H^{1}\left(T_{\mathcal{X} / S}\right)$ has constant rank. This was already noted in the first part of the proof.

\section{Codimension Formula}

Let $i: Y \hookrightarrow X$ be the inclusion of a Lagrangian subvariety in an irreducible symplectic manifold. In this section we show that if $Y$ has normal crossings, then the inclusion $M_{Y} \subset M_{Y}^{\prime}$ from Theorem 2.6 is an equality. In this way, we obtain a formula for the codimension of $M_{Y}$.

Lemma 4.1. Suppose $Y$ has normal crossings. Then

$$
\operatorname{ker}\left(H^{1}\left(\Omega_{X}\right) \stackrel{j^{*}}{\longrightarrow} H^{1}\left(\Omega_{\widetilde{Y}}\right)\right)=\operatorname{ker}\left(H^{1}\left(\Omega_{X}\right) \stackrel{i^{*}}{\longrightarrow} H^{1}\left(\widetilde{\Omega}_{Y}\right)\right),
$$

where $\nu: \tilde{Y} \rightarrow Y$ is the normalization.

Proof. As $j^{*}=\nu^{*} \circ i^{*}$ the inclusion $\supset$ is obvious. For the other direction it suffices to show that $\nu^{*}$ is injective on $\operatorname{im} i^{*}$. By Theorem 1.1 the subvariety $Y$ is projective, hence by [Del71, Del74] there is a functorial mixed Hodge structure on $H^{k}(Y, \mathbb{C})$ for every $k$. We denote by $F^{\bullet}$ the Hodge filtration on $H^{2}(Y)$ and by $W_{\bullet}$ the weight filtration. As a special case of [Le12, Cor 4.16], we deduce that

$$
H^{1}\left(\widetilde{\Omega}_{Y}\right)=\operatorname{Gr}_{F}^{1} H^{2}(Y)=F^{1} H^{2}(Y) / F^{2} H^{2}(Y) .
$$

Let $\ldots \rightrightarrows Y^{1} \rightrightarrows Y^{0} \rightarrow Y$ be the canonical semi-simplicial resolution in the simple normal crossing case, see e.g. [Le12, 4.8], or the one from Remark 3.3 in the normal crossing case. Note that $\widetilde{Y}=Y^{0}$. Consider the weight 
spectral sequence associated with the first graded objects of the Hodge filtration given by

$$
E_{1}^{r, s}=H^{s}\left(Y^{r}, \Omega_{Y^{r}}^{1}\right) \Rightarrow H^{r+s}\left(Y, \widetilde{\Omega}_{Y}^{1}\right)
$$

By [PS08, Thm $3.12(3)]$ it degenerates on the same level as the weight spectral sequence, which is known to degenerate at $E_{2}$. The differential $d_{1}: E_{1}^{0,1} \rightarrow E_{1}^{0,1}$ is given by $\delta: H^{1}\left(\Omega_{Y^{0}}\right) \rightarrow H^{1}\left(\Omega_{Y^{1}}\right)$ and degeneration at $E_{2}$ tells us that

$$
\begin{aligned}
\operatorname{Gr}_{2}^{W} \operatorname{Gr}_{F}^{1} H^{2}(Y) & =F^{1} H^{2}(Y) /\left(W_{1} F^{1} H^{2}(Y)+F^{2} H^{2}(Y)\right)=E_{\infty}^{0,1}=E_{2}^{0,1} \\
& =\operatorname{ker}\left(H^{1}\left(\Omega_{Y^{0}}\right) \rightarrow H^{1}\left(\Omega_{Y^{1}}\right)\right) .
\end{aligned}
$$

In other words, as $W_{2} \operatorname{Gr}_{F}^{1} H^{2}(Y)=\operatorname{Gr}_{F}^{1} H^{2}(Y)=H^{1}\left(\widetilde{\Omega}_{Y}\right)$ there is an exact sequence

$$
0 \rightarrow W_{1} \operatorname{Gr}_{F}^{1} H^{2}(Y) \rightarrow H^{1}\left(\widetilde{\Omega}_{Y}\right) \stackrel{\nu^{*}}{\longrightarrow} H^{1}\left(\Omega_{Y^{0}}\right) \rightarrow H^{1}\left(\Omega_{Y^{1}}\right),
$$

so that $\operatorname{ker} \nu^{*}=W_{1} \operatorname{Gr}_{F}^{1} H^{2}(Y)$. But the Hodge structure on $H^{2}(X, \mathbb{C})$ has pure weight two because $X$ is smooth. In particular, $W_{1} \operatorname{Gr}_{F}^{1} H^{2}(X)=$ 0 . Morphisms of mixed Hodge structures are strict with respect to both filtrations, so we have

$$
0=i^{*}\left(W_{1} \operatorname{Gr}_{F}^{1} H^{2}(X)\right)=\operatorname{im} i^{*} \cap W_{1} \operatorname{Gr}_{F}^{1} H^{2}(Y)=\operatorname{im} i^{*} \cap \operatorname{ker} \nu^{*}
$$

hence $\nu^{*}$ is injective on $\operatorname{im} i^{*}$ and we deduce $\operatorname{ker} i^{*}=\operatorname{ker} j^{*}$ completing the proof.

The following lemma generalizes [Vo92, Lem 2.3] to the normal crossing case.

Lemma 4.2. Suppose $Y$ has normal crossings. Then we have $T_{M_{Y}^{\prime}, 0}=$ $T_{M_{Y}, 0}$ for the Zariski tangent spaces at $0 \in M_{Y} \cap M_{Y}^{\prime}$.

Proof. By Lemma 2.5 the tangent space of $M_{Y}^{\prime}$ at 0 is

$$
T_{M_{Y}^{\prime}, 0}=\operatorname{ker}\left(j^{*} \circ \omega^{\prime}: H^{1}\left(X, T_{X}\right) \rightarrow H^{1}\left(\widetilde{\Omega}_{Y}\right)\right) .
$$

By Lemma 4.1 this equals $\operatorname{ker}\left(i^{*} \circ \omega^{\prime}: H^{1}\left(X, T_{X}\right) \rightarrow H^{1}\left(\Omega_{\widetilde{Y}}\right)\right)$, where $\widetilde{Y} \rightarrow$ $Y$ is the normalization. On the other hand, $M_{Y}$ is the smooth image of $p: \operatorname{Def}^{\mathrm{lt}}(i) \rightarrow M$ so that

$$
\begin{aligned}
T_{M_{Y}, 0} & =\operatorname{im}\left(p_{*}: T_{\operatorname{Def}^{\mathrm{lt}}(i), 0} \rightarrow T_{M, 0}\right) \\
& =\operatorname{im}\left(H^{1}\left(X, T_{\mathcal{X} / S}\langle\mathcal{Y}\rangle\right) \rightarrow H^{1}\left(X, T_{X}\right)\right) \\
& =\operatorname{ker}\left(H^{1}\left(X, T_{X}\right) \stackrel{\alpha}{\longrightarrow} H^{1}\left(Y, N_{Y / X}^{\prime}\right)\right)
\end{aligned}
$$

where the third equality holds because the sequence (3.3) is exact. 
By (3.1) and Proposition 3.6 we have a commutative diagram

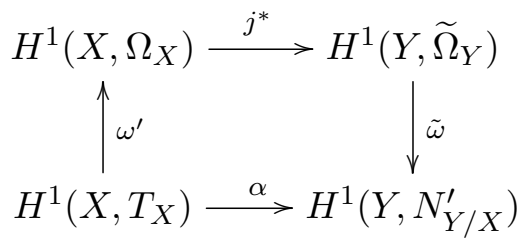

where the vertical maps are isomorphisms and this completes the proof.

Theorem 4.3. Let $i: Y \hookrightarrow X$ be a normal crossing Lagrangian subvariety in a compact irreducible symplectic manifold $X$, let $\nu: \widetilde{Y} \rightarrow Y$ be the normalization and denote $j=i \circ \nu$. Then $M_{Y}$ is smooth at 0 of codimension

$$
\operatorname{codim}_{M} M_{Y}=\operatorname{rk}\left(H^{2}(X, \mathbb{C}) \stackrel{j^{*}}{\longrightarrow} H^{2}(\widetilde{Y}, \mathbb{C})\right)
$$

in $M$.

Proof. By Theorems 2.6 and 3.8 we have $M_{Y} \subset M_{Y}^{\prime}$ and it suffices to show equality. This is deduced from $\operatorname{dim} M_{Y} \leq \operatorname{dim} M_{Y}^{\prime} \leq \operatorname{dim} T_{M_{Y}^{\prime}, 0}=$ $\operatorname{dim} T_{M_{Y}, 0}$, where the last equality comes from Lemma 4.2, again by invoking smoothness of $M_{Y}$.

Corollary 4.4. Let $K:=\operatorname{ker}\left(H^{2}(X, \mathbb{C}) \stackrel{j^{*}}{\longrightarrow} H^{2}(\widetilde{Y}, \mathbb{C})\right)$, let $q$ be the Beauville-Bogomolov quadratic form and consider the period domain

$$
Q:=\left\{\alpha \in \mathbb{P}\left(H^{2}(X, \mathbb{C})\right) \mid q(\alpha)=0, q(\alpha+\bar{\alpha})>0\right\}
$$

of $X$. Then the period map $\wp: M \rightarrow Q$ identifies $M_{Y}$ with $\mathbb{P}(K) \cap Q$ locally at $[X] \in M$.

Proof. As the period map identifies $M$ with $Q$ it suffices to show that $\wp\left(M_{Y}\right)=\mathbb{P}(K) \cap Q$. By [Huy99, 1.14], $\mathbb{P}(K) \cap Q$ is the locus where $K^{\perp} \subset H^{2}(X, \mathbb{C})$ remains of type $(1,1)$ and its codimension is $\operatorname{dim} K^{\perp}$. Note that $K^{\perp} \subset H^{1,1}(X)$ is defined over $\mathbb{Z}$ and therefore is spanned by the Chern classes of a collection of line bundles on $X$. By Lemma 3.4 the subspace $K^{\perp}$ remains of type $(1,1)$ over $M_{Y}$. Hence, $\wp\left(M_{Y}\right) \subset \mathbb{P}(K) \cap Q$. Moreover, we have

$$
\begin{aligned}
\operatorname{codim}_{Q} \wp\left(M_{Y}\right) & =\operatorname{codim}_{M} M_{Y}=\operatorname{rk}\left(j^{*}: H^{2}(X, \mathbb{C}) \rightarrow H^{2}(\widetilde{Y}, \mathbb{C})\right) \\
& =b_{2}(X)-\operatorname{dim} K=\operatorname{dim} K^{\perp} \\
& =\operatorname{codim}_{Q} \mathbb{P}(K) \cap Q .
\end{aligned}
$$

So both sets are equal. 


\section{Applications to Lagrangian fibrations}

In this section we give some applications of Theorems 2.6 and 4.3 to Lagrangian fibrations. Our main goal is to determine $\operatorname{codim}_{M} M_{Y}$. Let $X$ be an irreducible symplectic manifold. Recall that a Lagrangian fibration is a morphism $f: X \rightarrow B$ with connected fibers to a normal projective variety $B$ such that the general fiber of $f$ is a Lagrangian subvariety.

Lagrangian fibrations are an important tool to study irreducible symplectic manifolds. It is conjectured that an arbitrary irreducible symplectic manifold can always be deformed to one that admits a Lagrangian fibration. Moreover, Matsushita has shown in a series of papers [Mat99, Mat00, Mat01, Mat03] that every fibration of an irreducible symplectic manifold is a Lagrangian fibration and that the base $B$ resembles the projective space $\mathbb{P}^{n}$. The holomorphic Liouville-Arnol'd theorem shows that every smooth fiber is a complex torus, hence singular fibers enter the focus.

Hwang-Oguiso [HO09] classified generic singular fibers of a Lagrangian fibration. For such a fiber they defined the characteristic cycle, a (maybe infinite) cycle $\Theta$ of curves on the fiber, and they have shown that it is either a Kodaira singular fiber of an elliptic surface or an infinite chain of smooth rational curves intersecting transversally (so-called $I_{\infty}$-type). Locally, the fiber is isomorphic to $\Theta \times \mathbb{C}^{n-1}$ and the intersection graph of the fiber is a quotient of the graph of the characteristic cycle. The datum of the intersection graph of the fiber together with its local singularities is what we call fiber type.

In view of these classification results, Theorem 4.3 applies to the majority of (reductions of) generic singular fibers of a Lagrangian fibration. Only fibers with characteristic cycle of Kodaira types II, III and IV are not normal crossing varieties; for those we have Theorem 2.6. Here it is important that we consider locally trivial deformations. It entails that the fiber type in the Hwang-Oguiso sense does not change so that we obtain an invariant of this fiber type, see Theorem 5.7. Note that this is not in general the case for the characteristic cycle, see [HO10, Proposition 5.3] for an example.

5.1. Deforming fibrations. We show first that if we deform a fiber of a fibration then also the fibration deforms, see Lemma 5.2. Let $f: X \rightarrow B$ be a Lagrangian fibration and assume that $B$ is projective. Matsushita showed in [Mat09, Corollary 1.2] that there is a smooth hypersurface $\operatorname{Def}(X, f) \subset M$ with a relative Lagrangian fibration extending $f$

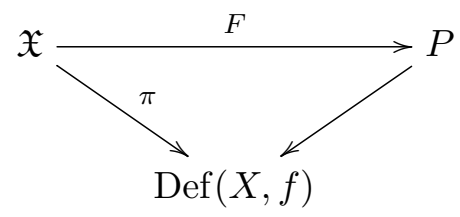


where $\pi: \mathfrak{X} \rightarrow \operatorname{Def}(X, f)$ is the restriction of the universal family to $\operatorname{Def}(X, f)$ and $P \rightarrow \operatorname{Def}(X, f)$ is a projective morphism. In particular, $F_{t}: \mathfrak{X}_{t} \rightarrow P_{t}$ is a Lagrangian fibration and $F_{0}=f$. Let $T$ be a smooth fiber of $f$ and let $M_{T} \subset M$ be as in Theorem 3.8. Then $M_{T}=\operatorname{Def}(X, f)$ by [Mat09, Proposition 2.1(3)]. The following lemma tells us that if the reduced fiber is preserved as a subvariety, then also the fibration is preserved.

Lemma 5.2. Let $f: X \rightarrow B$ be a Lagrangian fibration, let $t \in B$ and let $Y=\left(X_{t}\right)_{\text {red }}$ be the reduction of a fiber. Then we have $M_{Y} \subset \operatorname{Def}(X, f)$. Moreover, locally trivial deformations of $Y$ remain fibers.

Proof. By 5.1 it is sufficient to show $M_{Y} \subset M_{T}$. Let $Y=\cup_{i \in I} Y_{i}$ be a decomposition into irreducible components. As in (2.7) we have $M_{Y} \subset$ $\cap_{i} M_{\left[\sum_{i} n_{i} Y_{i}\right]}$ and for a smooth fiber $T$ of $f$ we have $\sum_{i} n_{i}\left[Y_{i}\right]=[T]$ and so that $M_{\left[\sum_{i} n_{i} Y_{i}\right]}=M_{[T]}=M_{T}$, where the last equality is Voisin's theorem. Put together this gives $M_{Y} \subset M_{T}=\operatorname{Def}(X, f)$. As Lagrangian fibrations are equidimensional, the last claim follows from the Rigidity Lemma [KM98, Lem 1.6].

5.3. Codimension estimates. Let $X$ be an irreducible symplectic manifold and let $f: X \rightarrow B$ be a Lagrangian fibration. We put $Y=\left(X_{t}\right)_{\text {red }}$ for $t \in D:=\left\{t \in B: X_{t}\right.$ is singular $\}$. The analytic subset $D$ is called the discriminant locus of $f$. We know by [Hwa08, Prop 4.1] and [HO09, Prop 3.1 ] that $D$ is nonempty and of pure codimension one.

Let $D_{0} \ni t$ be an irreducible component of $D$ and let $X_{0}:=X \times_{B} D_{0}=$ $f^{-1}\left(D_{0}\right)$. Let $Y=\cup_{i \in I} Y_{i}$ and $X_{0}=\cup_{j \in J} X_{j}$ be decompositions into irreducible components and consider the surjective map $j: I \rightarrow J$ mapping $i \in I$ to the unique $j=j(i) \in J$ with $Y_{i} \subset X_{j}$.

I am very grateful to Keiji Oguiso for explaining the following lemma.

Lemma 5.4. Let $f: X \rightarrow B$ be a Lagrangian fibration of a projective irreducible symplectic manifold $X$. Let $X_{0}=\bigcup_{j \in J} X_{j}$ where $J=\{1, \ldots, r\}$ and let $i: Y=\left(X_{t}\right)_{\text {red }} \hookrightarrow X$ for $t \in D_{0} \subset B$ be the reduction of a general singular fiber contained in $X_{0}$. Then

$$
\operatorname{rk}\left(H^{2}(X, \mathbb{C}) \stackrel{j^{*}}{\longrightarrow} H^{2}(\widetilde{Y}, \mathbb{C})\right) \geq r
$$

where $\nu: \tilde{Y} \rightarrow Y$ is the normalization and $j=\nu \circ i$. More precisely, the subspace of $H^{2}(X, \mathbb{C})$ generated by the classes of the divisors $X_{j}$ maps onto a subspace of dimension $\geq r-1$ not containing the class of the ample divisor.

Proof. Let $C \subset B$ be a curve obtained by the intersecting $n-1$ general very ample divisors on $B$ and consider the fiber product $X_{C}=X \times_{B} C$. As $B$ is normal, $X_{C}$ is smooth. As $t \in D_{0}$ is general, there is such a curve $C$ with 
$t \in C$. Let $H$ be a very ample divisor on $X$ and let $H_{1}, \ldots, H_{n-1} \in|H|$ be general. Then the intersection $S=X_{C} \cap H_{1} \cap \ldots \cap H_{n-1}$ is a smooth surface by Bertini's theorem. By construction it comes with a morphism $g: S \rightarrow C$.

Consider the diagram

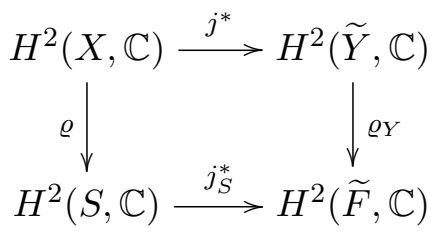

where $F=Y \cap H_{1} \cap \ldots \cap H_{n-1} \subset S$ and $\widetilde{F} \rightarrow F$ is the normalization. Note that $\widetilde{Y}$ is smooth by [HO09, Thm 1.3] and $\widetilde{F}$ is smooth, as $F$ is a curve. Let $Y=\bigcup_{i=1}^{s} Y_{i}$ and $F=\bigcup_{\lambda=1}^{q} F_{\lambda}$ be decompositions into irreducible components where $s=\# I$. We put $F(i):=Y_{i} \cap H_{1} \cap \ldots \cap H_{n-1}=\bigcup_{\lambda \in \Lambda_{i}} F_{\lambda}$, where $\Lambda_{i} \subset \Lambda:=\{1, \ldots, q\}$ is the subset of all $\lambda$ such that $F_{\lambda} \subset Y_{i}$. If the $H_{k}$ are general enough, $\Lambda$ is the disjoint union of the $\Lambda_{i}$.

We will show that the subspace $V \subset H^{2}(X, \mathbb{C})$ spanned by the $X_{j}$ and $H$ maps surjectively onto an $r$-dimensional subspace in $H^{2}(\widetilde{F}, \mathbb{C})$. This would imply the claim by diagram (5.1).

Write $X_{0}=\sum_{j} n_{j} X_{j}$ and $X_{t}=\sum_{i} n_{j(i)} Y_{i}$ as cycles, where as above $j(i)$ is the unique $j \in J$ with $Y_{i} \subset X_{j}$. Recall that $\Lambda=\coprod_{i} \Lambda_{i}$ is a disjoint union. So $n_{\lambda}:=n_{j(i)}$ for $\lambda \in \Lambda_{i}$ is well-defined and we have $F=\sum_{\lambda} n_{\lambda} F_{\lambda}$. As $F=\bigcup_{\lambda=1}^{q} F_{\lambda}$, we obtain $\widetilde{F}=\bigcup_{\lambda=1}^{q} \widetilde{F}_{\lambda}$ where $\widetilde{F}_{\lambda}$ is the normalization of $F_{\lambda}$. Thus,

$$
H^{2}(\widetilde{F}, \mathbb{C}) \cong \bigoplus_{\lambda=1}^{q} H^{2}\left(\widetilde{F}_{\lambda}, \mathbb{C}\right) \cong \mathbb{C}^{q} .
$$

If we denote the intersection pairing on $S$ by $(\cdot, \cdot)_{S}$, then under this isomorphism $j_{S}^{*}: H^{2}(S, \mathbb{C}) \rightarrow H^{2}(\widetilde{F}, \mathbb{C})$ is given by

$$
\alpha \mapsto\left(\left(\alpha, F_{1}\right)_{S}, \ldots,\left(\alpha, F_{q}\right)_{S}\right) .
$$

Let $\left\{x_{\lambda} \mid \lambda \in \Lambda\right\} \subset H^{2}(\widetilde{F}, \mathbb{C})^{\vee}$ be the dual basis of the basis of $H^{2}(\widetilde{F}, \mathbb{C})$ obtained corresponding to the standard basis of $\mathbb{C}^{q} \cong H^{2}(\widetilde{F}, \mathbb{C})$. By Zariski's Lemma [BHPV, Ch III, Lem 8.2] the subspace $W \subset H^{2}(S, \mathbb{C})$ spanned by the classes of the $F_{\lambda}$ maps surjectively to the hyperplane of $\mathbb{C}^{q}$ given by $\sum_{\lambda} n_{\lambda} x_{\lambda}=0$, So the subspace of $H^{2}(S, \mathbb{C})$ spanned by the classes of the $F_{\lambda}$ and $\left.H\right|_{S}$ maps surjectively onto $\mathbb{C}^{q}$. We have $\varrho_{Y}\left(j^{*} X_{j}\right)=j_{S}^{*} \varrho\left(X_{j}\right)=$ $\left(\left(\varrho\left(X_{j}\right), F_{\lambda}\right)_{S}\right)_{\lambda}$. As the $\Lambda_{i}$ are mutually disjoint, so are the $\Lambda_{j}:=\bigcup_{j(i)=j} \Lambda_{i}$. We see from $\left(\varrho\left(X_{j}\right), F_{\lambda}\right)_{S}=\sum_{\mu \in \Lambda_{j}}\left(F_{\mu}, F_{\lambda}\right)_{S}$ that the subspace of $H^{2}(X, \mathbb{C})$ generated by the $X_{j}$ surjects onto a subspace of $\mathbb{C}^{q}$ of dimension $\geq r-1$. The claim follows as the image of $V$ does not contain $j_{S}^{*}\left(\left.H\right|_{S}\right)$. 
Corollary 5.5. In the situation of the preceding lemma codim $M_{Y} \geq r$.

Proof. This follows from Theorem 2.6 and Lemma 5.4.

Note that there is no need for a normal crossing hypothesis in the corollary as we only prove an estimate and no equality. The codimension of $M_{Y}$ is thus bounded by the number of irreducible components of $X_{0}=f^{-1}\left(D_{0}\right)$ whereas the number of irreducible components of $Y$ does not a priori play a role. Hence, a very interesting and important question is the following

Question 5.6. Let $Y=\cup_{i \in I} Y_{i}$ and $X_{0}=\cup_{j \in J} X_{j}$ as in the beginning of section 5.3. Is then $\# I=\# J$ ? Do we always have $\operatorname{codim}_{M} M_{Y}=\# J$ for normal crossing $Y$ ?

There is no obvious reason, why these numbers should be equal, but in all examples we know they are equal. Recall that general singular fibers have been classified by Hwang-Oguiso according to their characteristic 1-cycle: this is an effective 1-cycle on a fiber $Y \subset X$, possibly an infinite sum of curves. It was shown to be of Kodaira type or of type $I_{\infty}$, see [HO09, Theorem 1.4] and [HO11, Theorem 2.4]. The type of a singular fiber will be the type of its characteristic 1-cycle.

Theorem 5.7. Let $X$ be an irreducible symplectic manifold and let $f: X \rightarrow$ $B$ be a Lagrangian fibration. Then $X$ can be deformed, keeping the fibration, to an irreducible symplectic manifold $X^{\prime}$ with a Lagrangian fibration $f^{\prime}$ : $X^{\prime} \rightarrow B^{\prime}$ such that outside a codimension 2 subset $Z \subset B^{\prime}$, all singular fibers of $f^{\prime}$ over the complement of $Z$ have a characteristic cycle of Kodaira type I, II, III or IV and such that for every irreducible component $D_{0}$ of the discriminant divisor the preimage $X_{D_{0}}^{\prime}=\left(f^{\prime}\right)^{-1}(0)$ is irreducible.

Proof. Let $D_{0}$ be an irreducible component of the discriminant divisor and let $Y=\left(X_{t}\right)_{\text {red }}$ for $t \in D_{0}$ be a general singular fiber. By Lemma 5.2, the space $M_{Y}$ is contained in $\operatorname{Def}(X, f)$. As the fiber type of a singular fiber is generically constant along an irreducible component of the discriminant divisor, it suffices to show that $M_{Y} \subsetneq \operatorname{Def}(X, f)$ if $X_{t}$ is not of type I-IV. But for all other Kodaira fibers, there are irreducible components of $X_{t}$ with different multiplicities. Fiber components with different multiplicities lie in different components of $X_{0}=f^{-1}\left(D_{0}\right)$, hence $\operatorname{codim} M_{Y} \geq 2$ by Corollary 5.5. By [Mat09, Corollary 1.2], codim $\operatorname{Def}(X, f)=1$ so that $M_{Y} \subsetneq \operatorname{Def}(X, f)$ and we conclude the proof.

Example 5.8. In the case of $\mathrm{K} 3$ surfaces, the situation becomes easier. For elliptic K3 surfaces it is not difficult to see that codim $M_{Y}=\# I=\# J$ of irreducible components of the reduction $Y$ of a fiber, if the latter has 
normal crossings, and codim $M_{Y} \geq \# I$ in all other cases, see [Le11, Thm VII.3.8]. The analogue of the Hodge-de Rham spectral sequence for $\widetilde{\Omega}_{Y}$ does not degenerate at $E_{1}$ if $Y$ does not have normal crossings, but one can show that for infinitesimal deformations $\mathcal{Y} \rightarrow S$ the $H^{q}\left(\widetilde{\Omega}_{\mathcal{Y} / S}^{p}\right)$ are free $\mathcal{O}_{S}$-modules if one uses the differentials in the spectral sequence. With this at hand, one deduces as in the normal crossing case that $\operatorname{Def}^{1 \mathrm{lt}}(i)$ and $M_{Y}$ are smooth. So $M_{Y} \subset M_{Y}^{\prime}$ in all cases. Consequently, using Theorem 5.7 any elliptic K3 surface can be deformed as a fibration to an elliptic K3 with only nodal and cuspidal curves as singularities. There are examples, where a cuspidal rational curve $Y$ deforms into two nodal curves so that we have $M_{Y} \subsetneq M_{Y}^{\prime}$.

5.9. Vista. There are several results assuming the general singular fibers to be of a special kind, see [HO10], [Saw08], [Saw12], [Thi08]. If we knew that complicated general singular fibers only show up in higher codimension in $M$, we could always deform to such special situations.

\section{REFERENCES}

[Bea83] Arnaud Beauville. Variétés Kähleriennes dont la première classe de Chern est nulle. J. Differential Geom., 18(4):755-782 (1984), 1983. - p. 6

[BHPV] Wolf P. Barth, Klaus Hulek, Chris A. M. Peters, and Antonius Van de Ven. Compact complex surfaces, Springer-Verlag, Berlin, second edition, 2004. p. 17

[Bog78] F. A. Bogomolov. Hamiltonian Kählerian manifolds. Dokl. Akad. Nauk SSSR, 243(5):1101-1104, 1978. - p. 1, 6

[Cam06] Frédéric Campana. Isotrivialité de certaines familles kählériennes de variétés non projectives. Math. Z., 252(1):147-156, 2006. - p. 4

[Del68] Pierre Deligne. Théorème de Lefschetz et critères de dégénérescence de suites spectrales. Inst. Hautes Études Sci. Publ. Math., (35):259-278, 1968. - p. 10, 11

[Del71] Pierre Deligne. Théorie de Hodge. II. Inst. Hautes Études Sci. Publ. Math., (40):5-57, 1971. - p. 12

[Del74] Pierre Deligne. Théorie de Hodge. III. Inst. Hautes Études Sci. Publ. Math., (44):5-77, 1974. - p. 12

[FK87] Hubert Flenner and Siegmund Kosarew. On locally trivial deformations. Publ. Res. Inst. Math. Sci., 23(4):627-665, 1987. - p. 6

[GHJ] M. Gross, D. Huybrechts, and D. Joyce. Calabi-Yau manifolds and related geometries. Universitext. Springer-Verlag, Berlin, 2003. - p. 6

[Fri83] R. Friedman. Global smoothings of varieties with normal crossings. Ann. of Math. (2), 118(1):75-114, 1983. - p. 9

[GLR14] Daniel Greb, Christian Lehn, and Sönke Rollenske. Lagrangian fibrations on hyperkähler fourfolds, Izvestiya: Mathematics, 78 (2014), no. 1. - p. 9

[GPR94] H. Grauert, Th. Peternell, and R. Remmert, editors. Several complex variables VII, Sheaf-theoretical methods in complex analysis, Springer-Verlag, Berlin, 1994. - p. 5 
[GR77] H. Grauert and R. Remmert. Theorie der Steinschen Räume, SpringerVerlag, Berlin, 1977. - p. 5

[Gra62] Hans Grauert. Über Modifikationen und exzeptionelle analytische Mengen. Math. Ann., 146:331-368, 1962. - p. 5

[HO09] Jun-Muk Hwang and Keiji Oguiso. Characteristic foliation on the discriminant hypersurface of a holomorphic Lagrangian fibration. Amer. J. Math., 131(4):981-1007, 2009. - p. 3, 15, 16, 17, 18

[HO10] Jun-Muk Hwang and Keiji Oguiso. Local structure of principally polarized stable lagrangian fibrations, 2010. preprint arXiv:1007.2043. - p. 15, 19

[HO11] Jun-Muk Hwang and Keiji Oguiso. Multiple fibers of holomorphic Lagrangian fibrations. Commun. Contemp. Math. 13 (2011), no. 2, 309-329. - p. 18

[Huy99] Daniel Huybrechts. Compact hyper-Kähler manifolds: basic results. Invent. Math., 135(1):63-113, 1999. - p. 14

[Hwa08] Jun-Muk Hwang. Base manifolds for fibrations of projective irreducible symplectic manifolds. Invent. Math., 174(3):625-644, 2008. - p. 16

[Kaw92] Yujiro Kawamata. Unobstructed deformations. A remark on a paper of Z. Ran: "Deformations of manifolds with torsion or negative canonical bundle". J. Algebraic Geom., 1(2):183-190, 1992. - p. 11

[Kaw97] Yujiro Kawamata. Erratum on: "Unobstructed deformations. A remark on a paper of Z. Ran: 'Deformations of manifolds with torsion or negative canonical bundle'”. J. Algebraic Geom., 6(4):803-804, 1997. - p. 11

[KM98] János Kollár and Shigefumi Mori. Birational geometry of algebraic varieties, Cambridge University Press, Cambridge, 1998. - p. 16

[Le11] Christian Lehn. Symplectic Lagrangian Fibrations. Dissertation, JohannesGutenberg-Universität Mainz, 2011. - p. 11, 19

[Le12] Christian Lehn. Normal crossing singularities and Hodge theory over Artin rings. 2012. preprint - p. 2, 3, 8, 9, 10, 12

[Mat99] Daisuke Matsushita. On fibre space structures of a projective irreducible symplectic manifold. Topology, 38(1):79-83, 1999. - p. 15

[Mat00] Daisuke Matsushita. Equidimensionality of Lagrangian fibrations on holomorphic symplectic manifolds. Math. Res. Lett., 7(4):389-391, 2000. - p. 15

[Mat01] Daisuke Matsushita. Addendum: "On fibre space structures of a projective irreducible symplectic manifold". Topology, 40(2):431-432, 2001. - p. 15

[Mat03] Daisuke Matsushita. Holomorphic symplectic manifolds and Lagrangian fibrations. Acta Appl. Math., 75(1-3):117-123, 2003. - p. 15

[Mat09] Daisuke Matsushita. On deformations of lagrangian fibrations. preprint arXiv:0903.2098. - p. 15, 16, 18

[PS08] Chris A. M. Peters and Joseph H. M. Steenbrink. Mixed Hodge structures, Springer-Verlag, Berlin, 2008. - p. 13

[Ra92Def] Ziv Ran. Deformations of manifolds with torsion or negative canonical bundle. J. Algebraic Geom., 1(2):279-291, 1992. - p. 2, 11

[Ra92Lif] Ziv Ran. Lifting of cohomology and unobstructedness of certain holomorphic maps. Bull. Amer. Math. Soc., 26(1):113-117, 1992. - p. 2

[Saw08] Justin Sawon. On the discriminant locus of a Lagrangian fibration. Math. Ann., 341(1):201-221, 2008. - p. 19

[Saw12] Justin Sawon. A finiteness theorem for Lagrangian fibrations. preprint arXiv: $1212.6470 .-$ p. 19 
[Saw15] Justin Sawon. Singular fibres of generic Lagrangian fibrations. unpublished manuscript. - p. 3,4

[Ser06] Edoardo Sernesi. Deformations of algebraic schemes, Springer-Verlag, Berlin, 2006. - p. 6, 12

[Thi08] Christian Thier. On the Monodromy of 4-dimensional Lagrangian Fibrations. Dissertation, Albert-Ludwigs-Universität Freiburg, 2008. - p. 19

[Tia87] Gang Tian. Smoothness of the universal deformation space of compact Calabi-Yau manifolds and its Petersson-Weil metric. In Mathematical aspects of string theory, pages 629-646. World Sci. Publishing, Singapore, 1987. - p. 1, 6

[Tod89] Andrey N. Todorov. The Weil-Petersson geometry of the moduli space of $\mathrm{SU}(n \geq 3)$ (Calabi-Yau) manifolds. I. Comm. Math. Phys., 126(2):325-346, 1989. - p. 1,6

[Vo92] Claire Voisin. Sur la stabilité des sous-variétés lagrangiennes des variétés symplectiques holomorphes. In Complex projective geometry, pages 294303. Cambridge Univ. Press, 1992. - p. 1, 2, 3, 7, 8, 13

[Vo1] Claire Voisin. Hodge theory and complex algebraic geometry. I, Cambridge University Press, Cambridge, 2002. - p. 8

[Vo2] Claire Voisin. Hodge theory and complex algebraic geometry. II, Cambridge University Press, Cambridge, 2003. - p. 7

Christian Lehn, Institut für Algebraische Geometrie, Gottfried Wilhelm Leibniz Universität Hannover, Welfengarten 1, 30167 Hannover, Germany

E-mail address: lehn@math.uni-hannover.de 